\title{
Available Transfer Capability Enhancement using Fruitfly Optimization
}

\section{Remmiya $\mathbf{R}$}

Department of Computer Science and Engineering,

Vins Christian Womens College of Engineering

Nagercoil, Tamil Nadu, India

Remmiya25@gmail.com

\author{
Brajula W \\ Department of Embedded System, \\ Udaya School of Engineering \\ Kanyakumari, Tamil Nadu, India \\ w.brajula@gmail.com
}

\begin{abstract}
In the recent decades, the FACTS devices are recognized as the proficient systems to fulfill the ever growing necessaries of transmission systems by adjusting it using the reconfigured models. Nevertheless, determining best sizing and placement for FACTS devices are results in the implementation at considerable expense, with respect to its efficiency. In order to accomplish the improved ATC, there is a need to evaluate the best location and its corresponding compensation levels which is achieved using fruit fly algorithm. This proposed fruit fly algorithm is compared over conventional GA algorithm to prove its efficiency for the betterment of ATC. Here, multiple TCSCs are implemented with varying scenarios using various IEEE benchmark standards IEEE 24 RTS, IEEE 30 and IEEE 57 bus systems with respect to evaluate the performance of enhanced ATC.
\end{abstract}

Keywords: Power system; ATC enhancement; fruit fly algorithm

\section{Introduction}

One of the significant circumstances of the competitive electricity market is the availability of power is open to all consumers to obtain power from the transmission system. This open access of power system network may produce the overload on the power system network more commonly [1]. Under deregulated environment, the substantial increase in power transfer is the important requirement [7][3]. Since Available Transfer Capability (ATC) gives the measure of the transfer capability of the existing transmission network for an increase in load to meet the commercial activities [5]. Except if the calculations of ATC are being used satisfactorily by the power transmission organization, more amount of power losses will occur in the power system network [2][4].

In general, the transfer capability means the capacity to transfer/increase/divert the power from one area/bus to another area/bus via existing system configuration [5][6]. Moreover, Several topological approaches for enhancing single/multi-area ATC presented Flexible AC Transmission System (FACTS) devices. In power system networks, FACTS devices are playing a vital role in efficiently controlling line power flows and achieving better voltage performance [8][9]. Three different classes of FACTS devices are combined shunt and series compensators, single shunt compensators and single series compensators [10][12]. The combined shunt and series compensators includes thyristor controlled voltage regulator (TCVR), thyristor-controlled phase-angle regulator (TCPAR) and unified power flow controller (UPFC). Single shunt compensators such as static var compensator (SVC) and STATCON, and single series compensators like a thyristor controlled series capacitor (TCSC) and thyristor controlled phase angle regulator (TCPAR) [13][17].

FACTS devices in a power system, provide economic benefits by postponing investment in the network. According to steady-state characteristic, FACTS devices operate by supplying or absorbing reactive power, increasing or reducing voltage profile and controlling the series impedance of transmission lines or phase angle. Mainly in [11][14] it is clear that the efficiency of these devices in the transmission system are intensely dependent on their location, number, size, and type. To improve the effectiveness of the ATC problem, the placement of FACTS controllers are to be optimal. Attaining an efficient Congestion management, while on enhancing Available Transfer Capability (ATC) of the 
network is achieved. Exceptional among the FACTS devices, thyristor controlled series compensator (TCSC) is infused with mysterious application potential in unambiguous controlling the power flow on the transmission line, venturing up transient robustness and damping power oscillations [15][18]. However, in deregulated power system, the optimal location of FACTS devices for maximizing the power transfer capability, numerous stochastic techniques like Particle Swarm Optimisation (PSO), Evolutionary Programming (EP), and Genetic Algorithms (GA) have been presented [16][19][21]. Among various approaches, Fruitfly Optimization Algorithm (FFO) is found to be an outstanding solution for optimization problems in power systems [3-4]. The FFO algorithm has many rewards such as a simple structure, immediately accessible for practical applications, the simplicity of implementation and speed to obtain solutions [20][22]. Therefore an efficient TCSC - FACTS device is modeled by power flow equations and optimally fix the FACTS parameters by FFO algorithm in such a way so as to enhance ATC in the deregulated electricity environment [23][24][25].

In the recent years, new artificial intelligence-based techniques have been proposed to design an FACTS-based supplementary damping controller and numbers of associated works are available in the literature by enhancing the available transfer capability of the power system. Here, we considered few of them. For improving the dynamic stability of power systems, the efficiency of the optimal location of UPFC has been examined by B. Vijay Kumar and N.V. Srikanth [26].

K. Bavithra et al. [27] investigated the research for the determination and enhancement of ATC by optimally set the FACTS parameters using particle swarm optimization-based methodology. In order to maximize power transfer transactions during normal and contingency situations Nireekshana et al. [28] presented the enhancement of ATC with the help of SVC and TCSC using Cat Swarm Optimization (CSO). Srikanta Mahapatra et al. [29] have suggested a hybrid Firefly Algorithm and Pattern Search (hFAPS) optimization based SSSC controller for damping power oscillations. A novel strategy for optimal placement of various FACTS controller and ATC enhancement in huge power systems have been brought in by M. Venkateswara Rao et al. [30]. For both single-machine infinite-bus and a multi-machine power system, Sidhartha Panda et al. [31] have offered HBFOA-PSO based optimal tuning algorithm is used to optimally and simultaneously tune the parameters of the PSS and SSSC-based damping controller.

M. Rashidinejad et al. have presented hybrid heuristic optimization technique include Real Genetic Algorithm (RGA) associated with analytical hierarchy process (AHP) and fuzzy sets to determine the optimum location and optimum capacity of TCSC in order to improve ATC as well as voltage profile [32].

\section{ATC Enhancement Model in Power System}

\subsection{Description on ATC}

In a deregulated power system structure, power producers and customers share a common transmission network for moving power from the point of generation to the point of consumption. All customers in this open access environment may try to generate the energy from the inexpensive source for better profit margin, which may lead to overloading and congestion of certain passages of the transmission network. This may cause the destruction of stability limits, line flow, voltage and thereby ruin the system security. Therefore these necessities need to determine sufficient "Available Transfer Capability" to guarantee that sustaining system reliability while serving a wide range of bilateral and multilateral transmissions. North American Electric Reliability Council (NERC) recognized a context for determining ATC of an interconnected network for a commercially feasible wholesale electricity market. In general, ATC is a measure of the transfer capability remaining in the physical transmission network for further commercial activity over and above already committed uses. Statistically, ATC refers to the Total Transfer Capability (TTC) minus the Transmission Reliability Margin (TRM), minus the sum of existing transmission commitments (which contains retail customer service) and the Capacity Benefit Margin (CBM).

Total Transfer Capability (TTC) defines the amount of portable electric power over the interconnected transmission lines by meeting pre- and post-contingency system conditions. Transmission Reliability Margin (TRM) is the maximum amount of transmission transfer capability necessary to ensure that the interconnected transmission network is secure under a reasonable range of uncertainties. Capacity Benefit Margin (CBM) refers to the amount of transmission transfer capability reserved by load serving entities to ensure access to generation from interconnected systems to meet generation reliability requirements.

Methods to compute ATC are well familiar. The classification of different schemes involves methods based on distribution factors and methods based on Continuation Power Flow (CPF). The method based on DC power transfer distribution factors employ DC power flow. Calculations by this method are fast 
and ease. However, it gives optimistic results. The AC power transfer distribution factor is simple, efficient and non-iterative to obtain ATC under bilateral and multilateral transmissions. CPF is a method for finding the maximum value of a scalar parameter in a linear function of variations in nodal injections at a set of buses. Originally presented for determining maximum laudability, CPF is malleable, without a change in principle, for other applications. The CPF algorithm successfully increases the controlling parameter in distinct steps and solves the resulting power flow problem at each step. It continuous the procedure until a given physical limit and encounters further increase. Moreover, CPF produces a solution even at voltage collapse points. A Continuation Power Flow is performed by starting from an initial point and then increasing the load by a factor up to some system limit is reached. The algorithm of ATC estimation by CPF describes in the following section.

\subsection{Mathematical Formulation of ATC}

Generally, ATC evaluation is a vital task that recently gained the interest of researchers and scholars to obtain enhanced developments toward the fields of Open access same time information system (OASIS). The typical description for ATC can be given as a metric to measure the transfer efficiency that available after the committed usage of physical transmission network which can be promoted for future commercial utility. The Eq. (1) gives the mathematical definition of ATC, in which, ETC ${ }_{E T C}$ specifies the Existing Transmission Commitments, $C B M_{C B M}$ represents the Capacity Benefit Margin, $T R M_{T R M}$ refers to the Total Transfer Capability, and $T T C_{T T C}$ denotes the Transmission Reliability Margin.

$$
A T C_{A T C}=T T C_{T T C} \quad T R M_{T R M} \quad\left(E T C_{E T C}+C B M_{C B M}\right)
$$

i. TTC: It is a measure that represents the amount of electric power that are transferred consistently using the interconnected transmission network so as to ensure the complete set of assured pre-contingency plus post-contingency system constraints.

ii. TRM: Similarly, TRM also define a measure that specifies the amount of transmission transfer ability necessary to guarantee the security among the interconnected transmission network when it is utilized under the circumstances with considerable set of uncertainties.

iii. CBM: As like the above, CBM is also the metric to denote the amount of transmission transfer ability that the load serving elements are employed to assure the access to generation of interconnected systems so as to gratify the generation consistency requests.

\section{Objectiive model on solving ATC enhancement problem}

\subsection{0bjective Model}

Consider a usual ATC bus that should be implemented for the betterment of ATC using minimum number of external resources i.e. the FACTS devices. Among all the models in FACTS, TCSCs are mainly focused in this paper so the objective to devise the enhancement of ATC corresponds to the implementations of TCSCs model. The resources are constrained based on the number of TCSCs implemented in the model.

$$
\left\lfloor L e_{t i}^{*}, X e_{t i}^{*}\right\rfloor=\underset{\left[L e_{t i}, X e_{t i}\right]}{\arg } \max A T C_{A T C} ; 0 \leq t i \leq N e_{T i} \quad 1
$$

Thus, the problem can be defined as given in Eq. (2), in which, $\left[L e_{t i}^{*}, X e_{t i}^{*}\right]$ defines the optimal connections in the set, $X e_{t i}$ and $L e_{t i}$ indicates the compensation level of $t i^{\text {th }}$ TCSC in the bus system, and the set of line indices respectively. Here, the main objective is to accomplish ATC betterment.

In order to achieve this objective, the proposed model intends to determine the best location and also the compensation level. Thus, all line indexes as well as the desired level of compensation are represented using a gene pair. Consider the chromosome as $S i_{p i}: 0 \leq p i \leq N i_{p i} \quad 1$ as well it is defined as given in Eq. (3), in which, $N i_{p i}$ refers to the size of population.

$$
S i_{p i}=\left\{s i_{0}, s i_{1}, \ldots, s i_{N i_{t i}}\right\}_{p i}
$$


Moreover, each specific gene available in a single chromosome $s i_{t i}$ is represented as a tuple $s i_{t i}=\{L e, X e\}_{t i}$, in which, Le specifies the line where the housing of TCSC is created. Moreover, Xe refers to the degree of compensation necessary for the TCSCs.

The estimation of fitness for $S i_{p i}$ is utilized as an iterative model used to formulate a portion of ATC evaluation procedure. The particular line index which represents the compensation level is indicated as $S i_{p i}$ is provided for the line data. Eventually, the value of $\lambda i$ is constant to the least potential value.

Assume the $\lambda i$ value for the first step as $\left(\lambda i=\lambda i_{I e}\right)$, in which, $\lambda i_{I e}=0.1$. Now, the compensation reactance is initialled then the continuous power flow evaluation is computed and record the bus voltages. If any case of voltage violations exists then the procedure is terminated. Here, the ATC evaluated after the termination of the procedure is considered as the fitness of the respective solutions. However, the procedure continues with respect to the $\lambda i$ values that are increasing for all iterations, till voltage violations exist. Thus, the upcoming sections present $\mathrm{s}$ the general formulations and its corresponding explanations.

\subsection{Fruitfly Algorithm}

Fruit fly algorithm is developed using the food searching behavior of fruit fly. Typically, it includes initialization, path construction, fitness function evaluation, and movement stage.

Initialization stage: Initially, the fruit flies are arbitrarily available in the search space as $I x$ and $I y$ as given in Eq. (4) and (5), in which, $R v$ specifies the arbitrary vector derived from uniform distribution.

$$
\begin{aligned}
& X i_{i}=X i+R v \\
& Y i_{i}=Y i+R v
\end{aligned}
$$

Path construction stage: The smell concentration and distance value for all fruit fly are defined in Eq. (6) and (7), in order, in which, Se specifies the smell concentration judgment value and $D i$ refers to the distance among the food location and the $i^{\text {th }}$ individual.

$$
\begin{aligned}
& D i=\sqrt{X i_{i}^{2}+Y i_{i}^{2}} \\
& S e=\frac{1}{D i}
\end{aligned}
$$

Fitness function evaluation stage: The Eq. (8) and (9) define the fitness, in which, sm represents the smell concentration of the single one, $\max (\mathrm{sm})$ denotes the maximal smell concentration between the fruit flies, $b s$ specifies the largest elements and $b i$ indicates the indices for various dimensions of smell vectors.

$$
\begin{aligned}
& s m=f n(\mathrm{Se}) \\
& b s, b i=\max (s m)
\end{aligned}
$$

Movement stage: Generally, fruit flies maintain keen sight regarding the location and optimal smell concentration value which are expressed in Eq. (10), (11) and (12), in order.

$$
\begin{aligned}
& s b=b s \\
& X i=X a(b i) \\
& Y i=Y a(b i)
\end{aligned}
$$

\subsection{Algorithmic Steps}

The proposed model is presented in the following steps. 
Step 1: (a) Read the system line data and bus data

System data: From bus, To bus, Line resistance, line reactance, half line charging, off nominal turns ratio, maximum line flows.

Bus data: $\quad$ Bus no, Bus type, $P_{g}, Q_{g}, P_{l}, Q_{l}, P_{m n}, P_{m x}, V_{s c}$ shunt capacitance data

(b) Cal $\quad P_{S}(i), Q_{s}(i)$, for $\mathrm{i}=1$ to $n$

where $\quad P_{s}(i)=P_{g}(i)-P_{l}(i)$

$$
Q_{S}(i)=Q_{g}(i)-Q_{l}(i)
$$

(c) Form $Y_{b}$ using sparsity technique

Step 2: (a) iter $=1$ iteration count $\quad$ Set $\left|\Delta P_{m x}\right|=0$ and $\left|\Delta Q_{m x}\right|=0$

(b) Calculate

$$
\begin{aligned}
& P_{c l}(i)=\sum_{q=1}^{n}\left|V_{i}\right|\left|V_{q} \| Y_{i q}\right| \cos \left(\begin{array}{ll}
\delta_{i q} & \theta_{i q}
\end{array}\right) \\
& Q_{c l}(i)=\sum_{q=1}^{n}\left|V_{i}\right|\left|V_{q} \| Y_{i q}\right| \sin \left(\delta_{i q}-\theta_{i q}\right)
\end{aligned}
$$

(c) Calculate

$$
\begin{aligned}
& P(i)=P_{s}(i)-P_{c l}(i) \\
& Q(i)=Q_{s}(i)-Q_{c l}(i) \text { for } \mathrm{i}=1 \text { to } n \\
& P_{s l k}=0.0, Q_{s l k}=0.0
\end{aligned}
$$

Set

(d) Calculate $\left|\Delta P_{m x}\right|$ and $\left|\Delta Q_{m x}\right|$ form $[\Delta P]$ and $[\Delta Q]$ vectors

(e) Is $\left|\Delta P_{m x}\right| \leq \epsilon$ and $\left|\Delta Q_{m x}\right| \leq \epsilon$

If yes

go to step 7, problem converged case

Step 3: $\quad$ Form Jacobian elements by initializing A matrix, formation of diagonal and off diagonal elements and then modifying jacobian elements with slack bus and generator buses finally create right hand side vector.

Step 4: $\quad$ Use Gauss-elimination method for solving $[A][\Delta X]=[B]$

Update the phase angle and voltage magnitudes

for $\mathrm{i}=1$ to $n$

$\delta_{i}=\delta_{i}+\Delta X_{i}$

$V_{i}=V_{i}+\left\{\Delta X_{i+n}\right\} V_{i}$

Step 5: $\quad$ One iteration is completed

Advance iteration count iter $=$ iter +1

If (iter > itermax)

go to step 2

Else

go to step 6

Step 6: NR not converged in "itermax" iterations

Step 7: $\quad$ NR converged in 'iter' iterations calculate: Line flows, Bus powers, slack bus power, Print the converged voltages, line flows and powers

Step 8: $\quad$ Read the sending bus (seller bus) $m$ and the receiving bus (buyer bus) $n$

Step 9: $\quad$ Assume some positive real power injection change $\Delta t p(=0.1)$, i.e. $\lambda$-factor at seller bus- $m$ and negative injection $\Delta t p(=0.1)$, i.e. $\lambda$-factor at the buyer bus- $n$ and form mismatch vector

Step 10: $\quad$ Repeat the load flow (i.e., from steps 2 to 7) and from the new line flows check whether any of the line is overloaded.

If yes else

stop the repeated power flow

go to step 9

Step 11: The maximum possible increment achieved above base-case load at the sink bus is the ATC

\section{Results and Discussions}

\subsection{Simulation Set up}

The performance of fruit fly is simulated using 3 benchmark bus systems, IEEE 24 RTS system, IEEE 30 bus system and IEEE 57 bus system. The betterment in ATC is comparing over conventional GA models. 
Here, the optimal outcome is accomplished by managing several TCSCs. In the previous work, two TCSCs were implemented to improve the ATC performance with respect to achieve best location and the compensation levels. In this paper, the simulation is implemented using five TCSCs devices. For each IEEE benchmark standard, the outcome for changing circumstances and the analytical evaluation is derived.

\subsection{Performance Anlaysis}

The best TCSC locations and its corresponding compensation levels using Fruit fly and GA are converse with one another. In this simulation the performance is evaluated using four TCSCs connections by fruit fly and GA for each IEEE benchmarks. However, implementing evolutionary models has the problem of local optimal solutions.

\subsection{Performance on IEEE 24 RTS System}

The optimal TCSC locations and its corresponding compensation levels using fruit fly and GA are presented in this section. In this IEEE benchmark standard, the proposed fruit fly model achieves $7 \mathrm{MW}$ more than GA for TCSC1, $8 \mathrm{MW}$ better than TCSC2, $8 \mathrm{MW}$ superior to GA for TCSC1, and $12 \mathrm{MW}$ more than GA for TCSC1 repectively. The Fig. 1 shows the simulation results of responses of fruit fly and GA for increasing TCSC connections using IEEE 24 RTS benchmark standard.

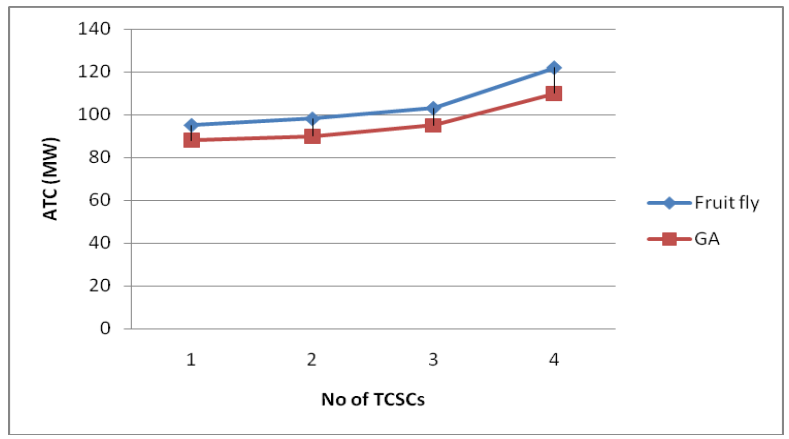

Fig. 1: Responses of fruit fly and GA for increasing TCSC connections using IEEE 24 RTS benchmark standard.

\subsection{Performance on IEEE 30 Bus System}

Similarly, the optimal TCSC locations and its corresponding compensation levels using fruit fly and GA are presented. In this IEEE benchmark standard, the proposed fruit fly model achieves 7 MW more than GA for TCSC1, 5 MW better than TCSC2, 3 MW superior to GA for TCSC1, and 5 MW more than GA for TCSC1 respectively. The Fig. 2 depicts the simulation of responses of fruit fly and GA for increasing TCSC connections using IEEE 30 bus system benchmark standard.

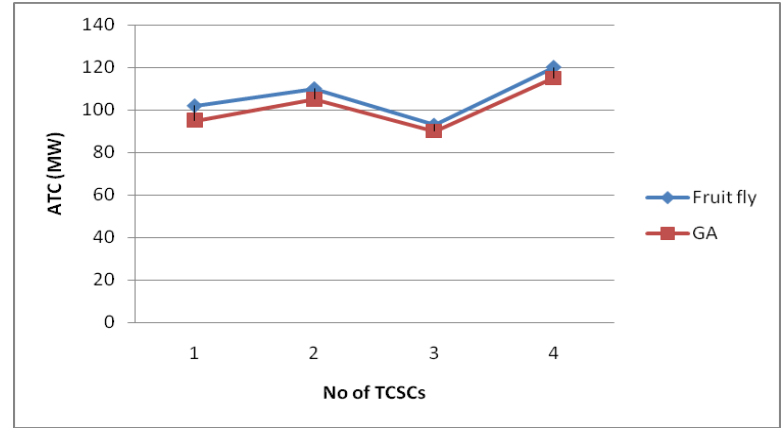

Fig. 2: Responses of fruit fly and GA for increasing TCSC connections using IEEE 24 RTS benchmark standard. 


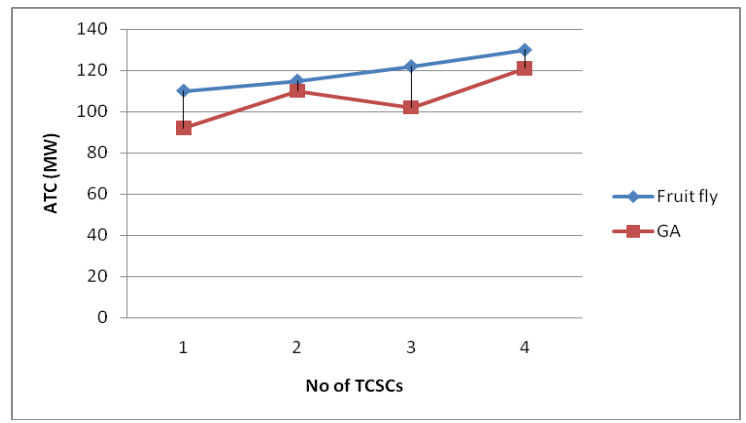

Fig. 3: Responses of fruit fly and GA for increasing TCSC connections using IEEE 24 RTS benchmark standard.

\subsection{Performance on IEEE 57 Bus Systems}

As like the above models, the best TCSC locations and its corresponding compensation levels using fruit fly and GA are presented here. In this IEEE benchmark standard, the proposed fruit fly model achieves 18 MW more than GA for TCSC1, 5 MW better than TCSC2, 20 MW superior to GA for TCSC1, and 19 MW more than GA for TCSC1 in order. The Fig. 3 illustrates the experimental outcome of responses of fruit fly and GA for increasing TCSC connections using IEEE 57 bus system benchmark standard.

\section{Conclusion}

This paper presents a clear and accurate evaluation on ATC using multiple TCSCs. The performance of fruit fly and GA was analysed to express the betterment of ATC using Fruit fly model. This simulation is implemented by incorporating response of ATC betterment with several TCSCs, sensitivity performance and statistics of performance. Initially, GA provides clear response for ATC betterment using the increased TCSCs connections. However, the performance for fruit fly is efficient and accurate toward the improvement of ATC that GA in terms of sensitivity and response. The statistics of the proposed model presents the betterment of ATC using fruit fly which is considerably improved than GA.

\section{Compliance with Ethical Standards}

Conflicts of interest: Authors declared that they have no conflict of interest.

Human participants: The conducted research follows the ethical standards and the authors ensured that they have not conducted any studies with human participants or animals.

\section{References}

[1] W. Li, Y. Hei, J. Yang and X. Shi, "Optimisation of non-uniform time-modulated conformal arrays using an improved non-dominated sorting genetic-II algorithm," IET Microwaves, Antennas \& Propagation, vol. 8, no. 4, pp. 287-294, 18 March 2014.

[2] Y. Chang, "Multi-objective optimal thyristor controlled series compensator installation strategy for transmission system loadability enhancement," IET Generation, Transmission \& Distribution, vol. 8, no. 3, pp. 552-562, March 2014.

[3] J. Liu and C. Chu, "Iterative Distributed Algorithms for Real-Time Available Transfer Capability Assessment of Multiarea Power Systems," IEEE Transactions on Smart Grid, vol. 6, no. 5, pp. 2569-2578, Sept. 2015.

[4] X. Tong, F. F. Wu and L. Qi, "Available Transfer Capability Calculation Using a Smoothing Pointwise Maximum Function," IEEE Transactions on Circuits and Systems I: Regular Papers, vol. 55, no. 1, pp. 462-474, Feb. 2008.

[5] S. Joo, C. Liu, Y. Shen, Z. B. Zabinsky and J. Lawarree, "Optimization techniques for Available Transfer Capability (ATC) and market calculations," IMA Journal of Management Mathematics, vol. 15, no. 4, pp. 321337, Oct. 2004.

[6] P. Du et al., "Probabilistic-Based Available Transfer Capability Assessment Considering Existing and Future Wind Generation Resources," IEEE Transactions on Sustainable Energy, vol. 6, no. 4, pp. 1263-1271, Oct. 2015.

[7] Y. Ou and C. Singh, "Calculation of risk and statistical indices associated with available transfer capability," IEE Proceedings - Generation, Transmission and Distribution, vol. 150, no. 2, pp. 239-244, March 2003.

[8] M. M. Othman and S. Busan, "A Novel Approach of Rescheduling the Critical Generators for a New Available Transfer Capability Determination," IEEE Transactions on Power Systems, vol. 31, no. 1, pp. 3-17, Jan. 2016.

[9] M. M. Othman and S. Busan, "A Novel Approach of Rescheduling the Critical Generators for a New Available Transfer Capability Determination," IEEE Transactions on Power Systems, vol. 31, no. 1, pp. 3-17, Jan. 2016. 
[10] J. W. Stahlhut and G. T. Heydt, "Stochastic-Algebraic Calculation of Available Transfer Capability," IEEE Transactions on Power Systems, vol. 22, no. 2, pp. 616-623, May 2007.

[11] A. Rajabi-Ghahnavieh, M. Fotuhi-Firuzabad, M. Shahidehpour and R. Feuillet, "Optimal Allocation of Available Transfer Capability in Operating Horizon," IEEE Transactions on Power Systems, vol. 24, no. 2, pp. 967-975, May 2009.

[12] H. Chen, X. Fang, R. Zhang, T. Jiang, G. Li and F. Li, "Available transfer capability evaluation in a deregulated electricity market considering correlated wind power," IET Generation, Transmission \& Distribution, vol. 12, no. 1, pp. 53-61, 212018.

[13] L. Hakim et al., "A Study on the Effect of Generation Shedding to Total Transfer Capability by Means of Transient Stability Constrained Optimal Power Flow," IEEE Transactions on Power Systems, vol. 24, no. 1, pp. 347-355, Feb. 2009.

[14] T. Jain, S. N. Singh and S. C. Srivastava, "Adaptive wavelet neural network-based fast dynamic available transfer capability determination," IET Generation, Transmission \& Distribution, vol. 4, no. 4, pp. 519-529, April 2010.

[15] N. A. Salim, M. M. Othman, M. S. Serwan, M. Fotuhi-Firuzabad, A. Safdarian and I. Musirin, "Determination of available transfer capability with implication of cascading collapse uncertainty," IET Generation, Transmission \& Distribution, vol. 8, no. 4, pp. 705-715, April 2014.

[16] L. Gang, C. Jinfu, C. Defu, S. Dongyuan and D. Xianzhong, "Probabilistic assessment of available transfer capability considering spatial correlation in wind power integrated system," IET Generation, Transmission \& Distribution, vol. 7, no. 12, pp. 1527-1535, December 2013.

[17] D. Rai, S. O. Faried, G. Ramakrishna and A. Edris, "Damping Inter-Area Oscillations Using Phase Imbalanced Series Compensation Schemes," IEEE Transactions on Power Systems, vol. 26, no. 3, pp. 1753-1761, Aug. 2011.

[18] P. K. Tiwari and Y. R. Sood, "An Efficient Approach for Optimal Allocation and Parameters Determination of TCSC With Investment Cost Recovery Under Competitive Power Market," IEEE Transactions on Power Systems, vol. 28, no. 3, pp. 2475-2484, Aug. 2013.

[19] L. D. Colvara, "The influence of the TCSC on power system transient energy," IEEE Latin America Transactions, vol. 3, no. 3, pp. 284-289, July 2005.

[20] L. Piyasinghe, Z. Miao, J. Khazaei and L. Fan, "Impedance Model-Based SSR Analysis for TCSC Compensated Type-3 Wind Energy Delivery Systems," IEEE Transactions on Sustainable Energy, vol. 6, no. 1, pp. 179-187, Jan. 2015.

[21] F. Bizzarri, A. Brambilla and F. Milano, "Analytic and Numerical Study of TCSC Devices: Unveiling the Crucial Role of Phase-Locked Loops," IEEE Transactions on Circuits and Systems I: Regular Papers, vol. 65, no. 6, pp. 1840-1849, June 2018.

[22] Y. Yang, X. Zhu, L. Gao, Y. Yuan and W. Yao, "Look-up table approaches for TCSC impedance control considering thyristor conduction characteristic," The Journal of Engineering, vol. 2017, no. 13, pp. 1408-1412, 2017.

[23] S. R. Joshi, E. P. Cheriyan and A. M. Kulkarni, "Output feedback SSR damping controller design based on modular discrete-time dynamic model of TCSC," IET Generation, Transmission \& Distribution, vol. 3, no. 6, pp. 561-573, June 2009.

[24] F. Wei, X. Lin, Z. Li, L. Chen and M. S. Khalid, "A New Distance Protection Method Considering TCSC-FCL Dynamic Impedance Characteristics," IEEE Transactions on Power Delivery, vol. 33, no. 3, pp. 1428-1437, June 2018.

[25] H. Kuang, L. Zheng, S. Li and X. Ding, "Voltage stability improvement of wind power grid-connected system using TCSC-STATCOM control," IET Renewable Power Generation, vol. 13, no. 2, pp. 215-219, 422019.

[26] B.Vijay kumar, and N.V.Srikanth, "Optimal location and sizing of unified power flow controller (UPFC) to improve dynamic stability: A hybrid technique”, International journal of Electrical Power and Energy Systems, vol. 64, pp.429-438, 2014.

[27] K Bavithra, SC Raja, and P Venkatesh, "Optimal setting of FACTS devices using particle swarm optimization for ATC enhancement in deregulated power system", IFAC, vol. 49, no. 1, pp 450-455, 2016.

[28] T.Nireekshana, G.Kesava Rao, and S.Sivanaga Raju, "Available transfer capability enhancement with FACTS using Cat Swarm Optimization", Ain Shams Engineering Journal, vol. 7, no. 1, pp 159-167, 2016.

[29] Srikanta Mahapatra, Sidhartha Panda, and Sarat Chandra Swain, "A hybrid firefly algorithm and pattern search technique for SSSC based power oscillation damping controller design", Ain Shams Engineering Journal, vol. 5, no. 4, pp 1177-1188, 2014.

[30] M.Venkateswara Rao, S.Sivanagaraju, and Chintalapudi V.Suresh, "Available transfer capability evaluation and enhancement using various FACTS controllers: Special focus on system security", Ain Shams Engineering Journal, vol. 7, no. 1, pp 191-207, 2016.

[31] Sidhartha Panda, Narendra Kumar Yegireddy, and Sangram Keshori Mohapatra, "Hybrid BFOA-PSO approach for coordinated design of PSS and SSSC-based controller considering time delays", International Journal of Electrical Power \& Energy Systems, vol. 49, pp 221-233, 2013.

[32] M. Rashidinejad, H. Farahmand, M. Fotuhi-Firuzabad, and A. A. Gharaveisi, "ATC enhancement using TCSC via artificial intelligent techniques", Electric Power Systems Research, vol. 78, no.1, pp. 11-20, Jan. 2008.

[33] Bo Xing, and Wen-Jing Gao, "Fruit Fly Optimization Algorithm" Innovative Computational Intelligence, pp 167170,2013

[34] John McCall, "Genetic algorithms for modelling and optimisation", Journal of Computational and Applied Mathematics, vol. 184, no. 1, pp 205-222, 2005. 